\title{
Anesthetic Considerations in Cardiac Patients Undergoing Neurosurgery
}

\author{
Rashmi Bhatt $^{1}$ Puneet Khanna ${ }^{2}$ \\ 1Department of Anaesthesia, Aberdeen Royal Infirmary, Aberdeen, \\ Scotland, United Kingdom \\ ${ }^{2}$ Department of Anaesthesiology, Pain Medicine and Critical Care, \\ All India Institute of Medical Sciences, New Delhi, India
}

Address for correspondence Puneet Khanna, MD Anesthesia, Department of Anaesthesiology, Pain Medicine and Critical Care, All India Institute of Medical Sciences, New Delhi 110048, India (e-mail: k.punit@yahoo.com).

J Neuroanaesthesiol Crit Care:2021;8:20-27

\begin{abstract}
Keywords

- anesthetic considerations

- cardiac disease

- neurosurgery

Elective and emergency neurosurgical procedures pose considerable risk in patients with cardiac disease. As the incidence of cardiac disease has increased in the general population over the past few decades, it is imperative to familiarize oneself with the challenges posed and the recommended management guidelines. The eventual outcome is significantly altered by the nature and severity of the underlying cardiac condition, as is the anesthetic management. A well-optimized cardiac condition can reduce morbidity and mortality considerably, which, however, may not be possible in neurosurgical emergencies. This review aims to look at various pathophysiological implications of cardiac disease, against the background of anesthetic management of neurosurgery. The discussion includes assessment and stratification of risk, appropriate investigations indicated, and the plan of anesthetic management. Even though no specific guidelines have been formulated, the discussion is largely to derive from the guidelines for patients with cardiac disease undergoing noncardiac surgery.
\end{abstract}

\section{Introduction}

Many neurosurgical procedures and associated pathophysiological states have significant interactions with the cardiovascular system in most healthy patients. Conversely, patients with underlying cardiac disease may come across the need to be anesthetized for neurosurgical procedures, which may be elective or emergency in nature. Several clinical considerations come into play for this group of patients, in terms of preoperative assessment and optimization, intraoperative management, and postoperative care. Preservation of cardiovascular stability is a primary concern, as the central nervous system (CNS) function depends on the presence of adequate oxygen and substrate for metabolism. This article attempts to review and discuss several aspects of neuroanesthesia for a patient with cardiovascular disease. This review is primarily narrative in character, with most of the recommendations having been referenced to the 2014 new ESC/ESA (European Society of Cardiology/European

Published online July 14, 2020
DOI https://doi.org/ $10.1055 / \mathrm{s}-0039-1692734$ ISSN 2348-0548.
Society of Anaesthesiology) guidelines on noncardiac surgery and the 2014 ACC/AHA (American College of Cardiology/ American Heart Association) guidelines on perioperative cardiovascular evaluation and management of patients undergoing noncardiac surgery. References have also been made to 2018 ESA guidelines for preoperative evaluation of adults undergoing elective noncardiac surgery. Apart from these, Ovid, PubMed, Google Scholar, and Medline were searched with MeSH terms "anesthetic management," "noncardiac surgery," and "neurosurgery," with a primary focus on review articles and controlled studies.

Preoperative cardiovascular disturbances are common in patients undergoing neurosurgical interventions that include, but are not limited to, blood pressure fluctuations, electrocardiographic abnormalities, arrhythmias, and myocardial ischemia or failure. ${ }^{1}$ There are concurrent central neurogenic effects on the heart as well as autonomic nervous system, as also the effects exerted due to underlying medical conditions. Preexisting cardiac disease, both symptomatic and 
asymptomatic, should be identified and investigated, as much as possible. The decision to perform further diagnostic evaluations should follow established guidelines, while customizing it as per patient and surgical indications. ${ }^{2}$ The use of appropriate scoring systems is a reliable and fairly standard way to stratify risk as well as predict outcome. These include the EuroScore, CAREscore, etc., which, however, are more useful in cardiac patients undergoing cardiac surgery. ${ }^{3,4}$ These unique issues pose a significant challenge to the anesthetist and therefore call for a systematic approach to the problem.

One is likely to come across patients in clinical practice with cardiac issues that may be congenital or acquired, such as hypertension, ischemic heart disease, valvular heart disease, arrhythmias, and patients with pacemakers or ICD (implantable cardioverter-defibrillator) in situ. - Table 1 summarizes common cardiac conditions and their relevance to the brain. The more critical ones may present in a decompensated state of a chronic cardiac illness. Deserving due attention is the list of many cardiac medications that these patients are often on and that could have an impact on the perioperative management. The discussion of management of most of these patients is based on the 2014 revised ACC/ AHA recommendations that provide a useful tool in terms of assessment, risk stratification, and optimization. Also taken into consideration are the 2014 new ESC/ESA guidelines on noncardiac surgery. ${ }^{5}$ The 2018 ESA guidelines on preoperative evaluation of adult patients undergoing noncardiac surgery also discuss the assessment and optimization of patients with cardiac disease. ${ }^{6}$ These have also been referred to and incorporated into this review.

\section{Preoperative Assessment and Optimization}

Preanesthetic evaluation is a process of clinical assessment, risk stratification, and optimization before surgery to reduce the perioperative morbidity and mortality. Most neurosurgical procedures are considered to be moderate to high-risk surgeries. Apart from these patient-related risk factors, each neurosurgical procedure carries its inherent risk

Table 1 Common cardiac conditions and their effects on the brain

\begin{tabular}{|l|l|}
\hline $\begin{array}{l}\text { Cardiac } \\
\text { pathology }\end{array}$ & CNS concerns \\
\hline Hypertension & Impaired cerebral microcirculation \\
\cline { 2 - 2 } & $\begin{array}{l}\text { White matter hyperintensities, } \\
\text { Alzheimer-like changes, lacunar infarcts }\end{array}$ \\
\cline { 2 - 2 } & Risk of aneurysmal rupture \\
\cline { 2 - 2 } & Risk of subarachnoid hemorrhage, stroke \\
\hline \multirow{2}{*}{$\begin{array}{l}\text { Valvular heart } \\
\text { disease }\end{array}$} & $\begin{array}{l}\text { Arrhythmias and risk of thromboembolism } \\
\text { leading to ischemic stroke }\end{array}$ \\
\cline { 2 - 2 } & Anticoagulation postvalvular replacement \\
\hline \multirow{2}{*}{$\begin{array}{l}\text { Congenital } \\
\text { heart disease }\end{array}$} & Risk of stroke and brain abscess \\
\cline { 2 - 2 } & Developmental delay in children \\
\hline Heart failure & Impaired cerebral perfusion \\
\hline Anticoagulants & Risk of hemorrhage/intracranial hematoma \\
\hline
\end{tabular}

Abbreviation: CNS, central nervous system. and requires specific preoperative evaluation and optimization. Risk stratification not only provides information to both the patient and the neurosurgeon to understand the benefit versus risk of each neurosurgical procedure but also helps the perioperative physician optimize the patient before surgery. The essential consideration in optimization is the urgency of indication. While the terms "emergency" and "urgency" are commonly used, the guideline writing committee for 2014 ACC/ AHA recommendations further defined two terminologies. ${ }^{4} \mathrm{~A}$ "time-sensitive procedure" defined as one in which a delay of 1 to 6 weeks, to allow for an evaluation and significant changes in management will negatively affect outcome, which would include most of so-called elective neurosurgical procedures. An "elective procedure" was defined as one in which the procedure could be delayed for up to 1 year, without significant consequences.

As many times as it may have been stated and restated, the significance of a detailed and structured history cannot be undermined. Combined with a thorough clinical examination, it is the simplest and the most effective tool in preoperative assessment. Apart from the duration and severity of the underlying cardiac disease, detailed information of the concomitant medications and any past medical interventions are also necessary. Two patients with similar cardiac conditions and planned for similar neurosurgical procedures may carry different preoperative risk, simply by being differently optimized. Besides a more accurate risk stratification, this step can also help decide the necessary investigations, thereby avoiding delays caused by unnecessary laboratory work. It goes without saying that these aspects may be rushed in case of an emergency, but none should be omitted or bypassed.

\section{Congenital Heart Disease}

As stated above, cardiac issues of significance could be a result of the underlying neurological condition or an independent entity. The latter, in turn, could either be a congenital or an acquired condition, both of which bear individual significance. Children and adults with CHD (congenital heart disease) are at increased risk of mortality and morbidity when undergoing noncardiac surgery. ${ }^{7}$ A point system for the risk stratification of a CHD patient before undergoing a procedure was developed by Mossad. ${ }^{8}$ This classification factors in variables such as complexity and physiology of cardiac lesion, type and urgency of surgery, age, and the duration of preoperative hospital stay. A patient presenting for noncardiac surgery may have undergone a corrective cardiac surgery, may have a residual cardiac lesion after correction, or may have an uncorrected lesion such as a grown-up with CHD (GUCHD). GUCHD patients represent a spectrum, from completely well patients with normal physiology to those with severely deranged physiology.

Cyanotic heart diseases such as tetralogy of Fallot predispose the patient to recurrent brain abscess as well as cerebral infracts due to underlying polycythemia, and also complicate the anesthetic management of the same. Fever, coagulopathy, dehydration, electrolyte imbalance, and metabolic acidosis are areas of concern, and most anesthetists prefer to have these corrected prior to surgery, where possible. Children surviving 
with dTGA (transposition of the great arteries) are also more likely to have abnormal neurological examinations, learning disabilities, and behavioral disorders, compared with general population..$^{9,10}$ Motor and global developmental delay may be seen more commonly in children with multiple types of CHD, in addition to TGA. ${ }^{10}$ More than one-half of newborns with CHD have clinical evidence of neurological abnormalities on examination even prior to corrective cardiac surgery, and these are a major risk factor for later neurodevelopmental impairment. ${ }^{11}$ Additional factors such as prolonged circulatory arrest or adverse events during cardiopulmonary bypass may add to the neurological insult. ${ }^{12}$

A thorough evaluation of the patient with CHD should be undertaken before elective neurosurgery, including an electrocardiogram (ECG), chest X-ray, echocardiography, and blood tests for full blood count and coagulation screen. When feasible, this should be performed in a center equipped with experienced surgeons and cardiac anesthesiologists. This ensures that CHD specialists are involved in close perioperative follow-up, and this should always be the case when a complex and cyanotic adult patient with CHD is prepared for neurosurgery.

Anesthetic management is centered around the balance between systemic and pulmonary vascular resistance, guiding the choice of anesthetic agents for induction and maintenance. Avoidance of activation of the sympathetic nervous system with consequent catecholamine release needs to be weighed against the effects of a reduction in systemic vascular resistance (SVR), which can worsen hypoxia, resulting in hemodynamic collapse. The cerebral perfusion pressure (CPP) may also be adversely impacted. Reactive pulmonary hypertension could be challenging to manage in older children or adults with untreated shunts, which may be worsened by sympathetic stimulation, acidosis, hypercapnia, and hypothermia. Input and output monitoring become even more significant, given the risk of heart failure weighed against hypoperfusion, which can aggravate underlying renal impairment, given the association with extracardiac anomalies of airway, skeletal, genitourinary, and gastrointestinal systems. Good pain relief and control of nausea-vomiting along with monitoring for events, such as dysrhythmias, bleeding, and thromboembolic events, are warranted.

\section{Ischemic Heart Disease and Heart Failure}

Active or unstable cardiac conditions, such as unstable angina, acute heart failure, and symptomatic cardiac arrhythmias, and valvular heart disease or recent myocardial infarction (MI) (within 6 months) are associated with very poor perioperative outcome. Heart failure is a major independent predictor of adverse perioperative outcome in noncardiac surgery and carries a greater perioperative risk than ischemic heart disease. ${ }^{13}$ Severely reduced left ventricular ejection fraction (LVEF < 30\%) itself is an independent contributor to perioperative outcome and a long-term risk factor for death in patients with heart failure undergoing elevated-risk noncardiac surgery. ${ }^{14}$ Diastolic dysfunction, with or without systolic dysfunction, also carries a significantly higher-risk postoperative heart failure. ${ }^{15}$ The role of a multidisciplinary team, with the anesthetist, neurosurgeon, cardiologist, possibly a cardiothoracic surgeon, and an intensivist, is reiterated.

For an elective procedure, the ideal approach would be to treat acute coronary episodes and optimize heart failure as much as possible. Appropriate medical therapy, if not already in place, should be initiated as well as titrated. This may include $\beta$-blockers, calcium channel blockers, antiarrhythmic drugs, diuretics, and possible use of ventricular assist devices. Almost all elective neurosurgical procedures can be delayed till the stabilization of an acute cardiac event.

\section{Valvular Heart Disease}

More challenging could be a situation with a patient with severe valvular heart disease but not in heart failure. In such cases, the risk of proceeding with a failing valve versus the benefit of having the valve replacement performed is a difficult decision to make. Severe valve stenosis can lead to adverse hemodynamic events leading to MI or heart failure with aortic stenosis (AS) and mitral stenosis (MS), respectively. Stenotic valvular lesions can be particularly challenging to manage intraoperatively. Induction in patients with AS should focus on stable hemodynamics while achieving adequate anesthetic depth, due to the low fixed cardiac output state. All efforts should be made to maintain normal sinus rhythm to ensure the atrial contribution to ventricular filing is maintained. Any hypotension can cause reduced coronary as well as cerebral perfusion pressure and should be rapidly managed with the early use of boluses of $\alpha$-adrenergic agonists. Anesthetic management of MS should focus on control of heart rate, ventricular preload, diminished right ventricular (RV) and left ventricular (LV) contractile function and coexisting pulmonary hypertension. All measures to avoid increases in pulmonary arterial (PA) pressures, by avoiding hypoxia, hypercarbia, acidosis, lung hyperexpansion, and nitrous oxide should be practiced. Patients are often on anticoagulation, whose management is discussed in detail, later in the text.

In patients ineligible for surgical valve replacements, the options of balloon dilatation or transcatheter valve replacement (for aortic valve) or valve commissurotomy (for mitral valve) should be considered. This decision making also should involve all the specialties, consider the urgency of neurosurgical indication (malignancy, aneurysm), and include the patient's opinion as well. The postoperative anticoagulation in valve replacements only adds to the challenges, with the potential to cause or worsen an intracranial bleed.

\section{Hypertension}

Patients with hypertension are probably the most common to be encountered. In many cases, newly diagnosed hypertensives are started on treatment, but insufficiently optimized due to lack of time or started on multiple medications in an attempt to bring down the "blood pressure within normal limits" urgently. Due consideration must also be given to the possibility of intracranial pathology as the cause of hypertension. Many of these patients end up getting either hypotensive due to overtreatment or have hypertensive 
surges intraoperatively. These are of particular significance in neuroanesthesia as blood pressure trends significantly impact CPP and therefore intracranial pressure (ICP). There is also a marked risk of acute postoperative hypertension, to the tune of $57 \%$ to $91 \%$ in patients undergoing intracranial neurosurgery. ${ }^{16}$ Rather than blindly target a "normal" number for blood pressure, it is reasonable to record a baseline and carry out a charting in whatever time is available. This chart should be used intraoperatively as a guide for blood pressure management, which will help preserve autoregulation as well as avoid unnecessary chasing of "normal values." It has been recommended to cancel elective surgery if the systolic blood pressure is $180 \mathrm{~mm} \mathrm{Hg}$ or higher or if the diastolic blood pressure is $110 \mathrm{~mm} \mathrm{Hg}$ or higher. ${ }^{17} \mathrm{~A}$ assessment of end-organ damage due to hypertension can be a useful guide to determine the urgency and need for treatment.

\section{Patients with Status Postcardiac Intervention}

This group includes patients having undergone corrective cardiac surgery or interventions for management of cardiac symptoms. The cardiac functional status for most patients would have been significantly improved, possibly optimized to the maximum. More relevant in these patients would be the management of concurrent medical treatment. The concerns and recommendations for continuation of antihypertensives, $\beta$-blockers, etc. have been discussed later in this text. The more challenging aspect is the management of anticoagulation. Patients having undergone valve replacement or at high risk of thrombosis would have been started on warfarin, whereas patients having required revascularization or thrombolytic therapy may have been started on single or dual antiplatelet therapy. Apart from these, newer anticoagulants such as rivaroxaban are also being used for long-term anticoagulation. Continuation of these may increase the risk of surgical bleeding, whereas discontinuation may exacerbate or complicate the underlying cardiac condition.

The decision to stop this therapy before surgery is controversial, and consultation with specialists such as the hematologist, cardiologist, and surgeon is useful. Contraindications to antithrombotic therapy include traumatic brain injury, craniotomy, hemorrhagic stroke, uncontrolled hypertension, and preexisting coagulopathies and/or advanced hepatic or renal disease. The urgency of procedure and the indication for starting the therapy in the first place should be reviewed. Patients on warfarin, especially for prosthetic valves in situ, are better managed with a bridging therapy to heparin, which is more titrable.

For patients at high risk of bleeding but also with high thrombotic risk, aspirin can be continued but all other antiplatelet medications must be withheld ( 7 days for clopidogrel and 14 days for ticlopidine), whereas if the thrombotic risk is low and if the bleeding risk outweighs the potential cardiovascular benefit, aspirin is withheld for 7 days as well. ${ }^{18}$ This is also recommended for patients undergoing spinal surgery or certain neurosurgical procedures. The decision should, however, be determined by individual assessment of risk versus benefit. ${ }^{6}$ This can be done using several criteria or risk factors for thromboembolism. ${ }^{19}$ Rivaroxaban needs to be withheld for at least 48 hours to eliminate its risk of bleeding, and it is generally restarted 48 to 72 hours postoperatively, thus eliminating the need for bridging therapy. Patients with coronary stents (bare metal as well as drug eluting) need individual risk stratification for continuation or stopping of antiplatelet drugs. ${ }^{20}$ The use of vitamin $\mathrm{K}$, fresh frozen plasma, and prothrombin complex concentrate should be considered as appropriate.

\section{Preoperative Medications}

Patients diagnosed with long-term, optimized hypertension should avoid changes in the regimen, in the immediate preoperative period, if possible, unless essential. Although ACC/AHA guidelines recommend continuation of angiotensin-converting enzyme (ACE) inhibitors and angiotensin receptor blockers, ${ }^{4}$ it may be reasonable to withhold them on the day of surgery, given the risk of protracted hypotension, which is far more ominous in neuroanesthesia. Patients on diuretics need extra caution when being administered osmotic diuretics such as mannitol, and an eye should be kept on intravascular volume as well as electrolytes. The accompanying diuresis may result in volume contraction, which increases the risk of hypotension causing myocardial or cerebral ischemia. These acute changes in intravascular volume may not be tolerated well by patients with decreased cardiac reserve.

Multiple studies have reported improved outcome in patients receiving perioperative $\beta$-blockers; however, newer studies have reported that perioperative $\beta$-blockers may not be effective if heart rate is not well controlled or in low-risk patients. ${ }^{21}$ The recommendations on perioperative $\beta$-blockade have been seriously challenged after the discovery of scientific unreliability in the DECREASE (Dutch Echocardiographic Cardiac Risk Evaluation Applying Stress Echocardiography) studies that provided much of the evidence in its support. ${ }^{22}$ The evidence in this regard has been critically reanalyzed, and now the only remaining $1 \mathrm{~B}$ recommendation is that patients currently on this therapy should continue it during the perioperative period. ${ }^{6}$

In a recent retrospective review of patients for noncardiac surgery, acute surgical anemia increased the risks of cardiac complications in $\beta$-blocked patients, hence suggesting that higher transfusion triggers should be considered in elective surgical patients on $\beta$-blockers. ${ }^{23}$ The current 2014 ACC/AHA guidelines on the perioperative $\beta$-blocker administration advocate that perioperative $\beta$-blockade should be used in patients on $\beta$-blockers and those with positive stress test undergoing major vascular surgery ${ }^{24}$ Due consideration should be given to severe bradycardia, in $\beta$-blocked patients, which may occur in cases of herniation. Acute administration of $\beta$-blockers without titration has been concluded to be unfavorable for outcome, whereas statins have been shown to improve perioperative cardiac outcome, hence are continued in patients currently taking them. ${ }^{25}$

Very careful risk-benefit analysis is called for, in patients on long-term anticoagulation, for several conditions, for example, AF, deep vein thrombosis, valve replacement, or coronary stenting. This has been discussed in detail earlier in the text. 


\section{Investigations and Cardiac Testing}

Preoperative investigations should include cardiac testing (as indicated) as well as the general investigations. Most patients undergoing neurosurgery will need a hemoglobin testing and a blood group and cross-match (where transfusion is a possibility). Other blood investigations should be governed by presence of specific conditions such as renal function tests in diabetes or long-standing hypertension and electrolytes in patients on diuretics or mannitol. A resting ECG is desirable in all major procedures.

Preoperative cardiac testing incorporates functional testing as well as investigations. Functional tests are used to obtain information that may have been missing or insufficient in history. Functional status is a reliable predictor of perioperative and long-term cardiac events, and so patients with reduced functional status preoperatively are at an increased risk of complications. On the other hand, a good functional status in an asymptomatic patient can rule out the need for several investigations. The most common method of functional assessment is metabolic equivalents (METs), in which 1 MET is the resting or basal oxygen consumption of a 40-year-old, 70-kg man and is largely derived from activities of daily living. ${ }^{26}$ Perioperative cardiac and long-term risks are increased in patients unable to perform 4 METs of work during daily activities. More formally applied scales such as the DASI (Duke Activity Status Index) and the Specific Activity Scale can be used to assess the functional status. ${ }^{27,28}$ It may be noted here that neurosurgical patients may have a limited functional status by virtue of underlying pathology as well, and this must be borne in mind during evaluation. The 2018 ESA guidelines recommend the use of the ACS National Surgical Quality Improvement Program (NSQIP) index score and the RCRI (Revised Cardiac Risk Index) in conjunction, for risk stratification of preoperative cardiac risk. ${ }^{29}$

As mentioned above, a resting preoperative 12-lead ECG is required for almost all major procedures. It is particularly useful as a baseline, to compare with in case of any new changes in the postoperative period. It is preferable to obtain one within last 3 months, if the patient is stable. It must be noted that some ECG changes may be a result of the underlying neurological issue rather than a cardiac one. For example, a patient with subarachnoid hemorrhage (SAH) may show several rhythm abnormalities on ECG. ECG abnormalities occur in $50 \%$ to $72 \%$ of patients with intracranial pathology, some of which may not appear for 2 weeks after the first insult but are not always associated with cardiac abnormalities. ${ }^{30}$ Most common findings are ST-segment changes, flat or inverted $\mathrm{T}$ waves, prominent $\mathrm{U}$ waves, and prolongation of the QTc interval. ${ }^{31}$ Therefore, the extent of myocardial ischemia is difficult to assess from ECG alone in a patient with intracranial pathology.

A preoperative LV function evaluation with echocardiography should be asked for a patient with undiagnosed dyspnea, or worsening heart failure, or any other change in clinical status. Postvalvular replacement should also be requested, if more than 1 year has elapsed since the last evaluation. ${ }^{32}$ In patients with valvular heart disease, severity of stenosis or regurgitation, systolic function, and right heart pressures should all be estimated. If time permits, a stress echo can provide more accurate and reliable information. If further indicated, nuclear imaging study or coronary angiography may be performed as appropriate.

The measurement of the serum concentrations of brain natriuretic peptide (BNP) or its inactive precursor N-terminal pro-B-type natriuretic peptide (NT-proBNP) may be used for risk stratification and prognostication. ${ }^{33}$ The assessment of cardiac troponins is recommended in high-risk patients both before and 48 to 72 hours after surgery. ${ }^{5}$ Measurement of biomarkers, especially natriuretic peptides, may be helpful in assessing and diagnosing patients with heart failure. However, there is no evidence for their routine use in patients with cardiac disease.

\section{Anesthetic Management}

Basic principles of maintaining hemodynamic stability and cerebral protection apply as for neurosurgical procedures performed in any patient. However, the use of goal-directed fluid and drug therapies, along with invasive or noninvasive cardiac output monitoring to improve outcome, has found support in many studies. The delicate balance between maintaining a relative hypertensive state and preventing hypertensive surges becomes even more challenging against the background of cardiac disease. On occasion, the need to maintain a low normal blood pressure, as during arteriovenous malformation repair, or inducing hypertension to maintain cerebral perfusion during vasospasm, following SAH, adds to the anesthetic challenges. These hemodynamic changes can have a profound effect on an already compromised cardiovascular system.

A good, balanced anesthetic technique is crucial for cerebral protection, preserving CPP, preventing surges in ICP, reducing cerebral metabolic rate of oxygen consumption $\left(\mathrm{CMRO}_{2}\right)$, and preventing secondary insults. Furthermore, the anesthetic technique of choice should maintain or only minimally interfere with cerebral autoregulation and responsiveness to $\mathrm{CO}_{2}$. It should also be able to maintain relaxation of the brain and provide fast, predictable recovery for early evaluation of the surgery.

Of particular concern is the delicate management of fluid balance. Patients with cardiac disease, especially heart failure and valvular heart disease, are at increased risk of fluid overloading. This should especially be taken care of in emergencies such as traumatic brain injury or SAH, where an attempt of fluid resuscitation can cause cardiac compromise. The use of agents such as mannitol and furosemide, used to decrease ICP, can cause fluid and electrolyte imbalance. Particularly $\mathrm{K}^{+}$depletion can precipitate arrhythmias in susceptible patients and cause problems in patients on treatment with digoxin.

Goals during induction of anesthesia are to maintain cerebral and myocardial perfusion with judicious use of appropriate induction agents. Both propofol and thiopentone preserve cerebral autoregulation and decrease metabolic requirement of $\mathrm{O}_{2}$ as well as cerebral blood flow. ${ }^{34}$ Generous 
doses of opioids at induction help in preventing exaggerated hemodynamic responses to airway management. The prophylactic use of vasopressors may be considered to avoid extreme changes in hemodynamics; the choice of appropriate agent should be made with the consideration of underlying cardiac condition. This may include the use of drugs such as dobutamine, noradrenaline, and adrenaline to maintain cardiac output as well as SVR. Even though mean blood pressure, and therefore CPP, is increased by all catecholamines, adrenaline has been seen to significantly increase the cerebral blood flow, compared with noradrenaline and dopamine, in some animal studies. ${ }^{35}$ The use of total intravenous anesthesia, with monitoring of the depth of anesthesia, has become a standard practice in many centers, due to a predictable and consistent induction as well as maintenance of anesthesia. The use of target-controlled infusion offers the benefit of use of optimum doses of hypnotic agents with stable hemodynamics. It has especially become the technique of choice for traumatic brain injury. The choice of muscle relaxants is dictated by the need for electrophysiological monitoring during the surgery. Intraoperative electrophysiological monitoring may help prevent postoperative deficits.

The maintenance of anesthesia can be performed using volatile as well as intravenous anesthetics. Apart from the neuroanesthetic perspective, the use of either is acceptable in patients with cardiac disease. The use of total intravenous anesthesia (TIVA) offers the advantage of more accurate titration of anesthetic agents and hence better control of hemodynamics. The use of nitrous oxide is better avoided against the background of pulmonary hypertension and in conjunction with volatile agents when motor-evoked potentials are being monitored. ${ }^{36}$

Apart from standard monitoring, advanced cardiac monitoring may be considered. As stated earlier, ECG interpretation can be tricky in these patients due to interplay of cardiac as well as neurological factors. Common ECG changes include T-wave abnormalities, Q-T prolongation (which may predispose patients to torsades de pointes and ventricular fibrillation), ST segment changes, prominent U waves, and various rhythm abnormalities, such as supraventricular tachyarrhythmias, bradyarrhythmias, and ventricular tachyarrhythmias. ${ }^{37}$ These should be treated with $\beta$-blockers, calcium channel blockers, and other antiarrhythmic drugs, as indicated. The mechanism of these changes is understood to be multifactorial, which include elevated catecholamine levels, hypercortisolism, and hyperkalemia. There is also a strong association between hypothalamic lesions and myocardial damage. ${ }^{38}$

The use of transesophageal echocardiography (TEE) is especially beneficial in patients with cardiac disease, as early changes in myocardial function may be detected. Invasive blood pressure monitoring is recommended in most patients, governed by either the underlying cardiac condition or the neurosurgical procedure planned. The monitoring of cardiac output (along with stroke volume, stroke volume variation, and SVR) may add significant clinical input to the management. Transcranial Doppler, in particular, is useful to determine whether autoregulation is intact and whether a protocol is appropriate for maintaining CPP.
Positioning for surgery may also bear implications that are more serious than for normal patients. Care must be taken of the change in hemodynamics during positioning, especially sitting up, prone, etc. The sitting position for craniotomy may be required in certain neurosurgical procedures. Problems associated with this position, however, limit its use in patients with cardiac disease. It is not uncommon to observe severe decreases in blood pressure in the sitting position with anesthetized patients. In patients with coronary artery disease, this could result in myocardial ischemia, whereas in patients with valvular heart diseases requiring maintenance of high preload, cardiovascular collapse may occur. ${ }^{39}$ Thus, the sitting position may be avoided in these patients, where possible. And even when used, vigilant monitoring with invasive cardiac output monitoring and TEE is indicated.

The consideration of stable hemodynamics with minimal surges is applied during emergence as well. The continuation of remifentanil infusion till the end serves this purpose well. Dexmedetomidine has also been found to be a useful component of balanced anesthesia; however, recommendations and evidence for the use of same are awaited. Potential advantages of neuroprotection, preservation of neuronal function, cardiovascular stability, opioidsparing effects, and minimal respiratory depression during awake procedures and intensive care sedation render it a useful anesthetic adjunct. ${ }^{40}$

Postoperative pain management is an important consideration to prevent tachycardia and increased myocardial oxygen demand, while also preventing surges in ICP. Multimodal analgesia, including the use of patient- controlled analgesia, with acetaminophen and opioids, as well as surgical infiltration with local anesthetic agents is useful. Solution containing adrenaline, to reduce bleeding, should be avoided or used with caution, due to the risk of arrhythmias. Attention must also be paid to treatment of postoperative nausea and vomiting (PONV). Where postoperative mechanical ventilation is continued, the decision to extubate must factor in the underlying cardiac disease. Effective management of pain and PONV and use of shortacting opioids can help fast-track extubation and prevent adverse outcome.

The use of postoperative intensive care, where indicated, is a good measure to improve outcome. Patients having undergone neurosurgical procedures have been reported to develop stress or Takotsubo's cardiomyopathy, due to catecholamine surge. Intensive care management should be multidisciplinary, with a considerable contribution of cardiology team. The continuation of cardiac medication should be attempted as much as possible and resumption of anticoagulation considered, where and when feasible. In cases in which the surgery was performed as an emergency procedure, detailed cardiac evaluation should be requested as soon as feasible.

Cardiac emergencies occurring intraoperatively could be challenging to manage. The emergency could be due to the underlying pathology or the neurosurgical procedure. These could range from arrhythmias and hemodynamic instability to cardiac arrest. Cardiopulmonary resuscitation (CPR) could be particularly challenging due to positioning, like the 
sitting or the prone position. Procedures such as craniotomy for tumors, epilepsy surgery, and skull base surgery have reported incidences of bradycardia and asystole, whereas posterior fossa surgery has been shown to result in ST elevation, ventricular fibrillation, and asystole. CPR has been successfully applied in lateral and sitting position; however, there is lack of standard guidelines related to management of patients in these positions. ${ }^{41}$ Most patients respond to removal of surgical stimulus (scalp traction), atropine, and resuscitation. ${ }^{42}$

\section{Conclusion}

Patients with cardiac disease are at risk of significant adverse cardiac events while undergoing neurosurgical procedures, elective as well as emergency. A careful evaluation of risk factors, with the best possible optimization of concurrent illness, is the most reasonable plan. The involvement of a multidisciplinary team should be the standard practice.

Conflict of Interest

None declared.

\section{References}

1 Hedge J, Balajibabu PR, Sivaraman T. The patient with ischaemic heart disease undergoing non cardiac surgery. Indian J Anaesth 2017;61(9):705-711

2 Kaul TK, Tayal G. Anaesthetic considerations in cardiac patients undergoing non cardiac surgery. Indian J Anaesth 2007;51:280-286

3 Kristensen SD, Knuuti J. New ESC/ESA guidelines on non-cardiac surgery: cardiovascular assessment and management. Eur Heart J 2014;35(35):2344-2345

4 Fleisher LA, Fleischmann KE, Auerbach AD, et al. 2014 ACC/ AHA guideline on perioperative cardiovascular evaluation and management of patients undergoing noncardiac surgery: executive summary: a report of the American College of Cardiology/American Heart Association Task Force on Practice Guidelines. Circulation 2014;130(24):2215-2245

5 Kristensen SD, Knuuti J, Saraste A, et al; Authors/Task Force Members. 2014 ESC/ESA guidelines on non-cardiac surgery: cardiovascular assessment and management: the Joint Task Force on non-cardiac surgery: cardiovascular assessment and management of the European Society of Cardiology (ESC) and the European Society of Anaesthesiology (ESA). Eur Heart J 2014;35(35):2383-2431

6 De Hert S, Staender S, Fritsch G, et al. Pre-operative evaluation of adults undergoing elective noncardiac surgery: updated guideline from the European Society of Anaesthesiology. Eur J Anaesthesiol 2018;35(6):407-465

7 Baehner T, Ellerkmann RK. Anesthesia in adults with congenital heart disease. Curr Opin Anaesthesiol 2017;30(3):418-425

8 Howard-Quijano K, Smith M, Schwarzenberger JC. Perioperative care of adults with congenital heart disease for non-cardiac surgery. Curr Anesthesiol Rep 2013;3:144-150

9 Limperopoulos C, Majnemer A, Shevell MI, Rosenblatt B, Rohlicek C, Tchervenkov C. Neurologic status of newborns with congenital heart defects before open heart surgery. Pediatrics 1999;103(2):402-408

10 Ellerbeck KA, Smith ML, Holden EW, et al. Neurodevelopmental outcomes in children surviving d-transposition of the great arteries. J Dev Behav Pediatr 1998;19(5):335-341
11 Limperopoulos C, Majnemer A, Shevell MI, et al. Predictors of developmental disabilities after open heart surgery in young children with congenital heart defects. J Pediatr 2002;141(1):51-58

12 Mahle WT, Tavani F, Zimmerman RA, et al. An MRI study of neurological injury before and after congenital heart surgery. Circulation 2002;106(12, Suppl 1):I109-I114

13 Hammill BG, Curtis LH, Bennett-Guerrero E, et al. Impact of heart failure on patients undergoing major noncardiac surgery. Anesthesiology 2008;108(4):559-567

14 Goldman L, Caldera DL, Nussbaum SR, et al. Multifactorial index of cardiac risk in noncardiac surgical procedures. N Engl J Med 1977;297(16):845-850

15 Healy KO, Waksmonski CA, Altman RK, Stetson PD, Reyentovich A, Maurer MS. Perioperative outcome and long-term mortality for heart failure patients undergoing intermediate- and high-risk noncardiac surgery: impact of left ventricular ejection fraction. Congest Heart Fail 2010;16(2):45-49

16 Horwitz NH, Rizzoli HV. Postoperative Complications of Intracranial Neurological Surgery. Baltimore, MD: Williams \& Wilkins;1982;12-20:111-132

17 Jellish WS. Anesthetic issues and perioperative blood pressure management in patients who have cerebrovascular diseases undergoing surgical procedures. Neurol Clin 2006; 24(4):647-659,viii

18 Llau JV, Lopez-Forte C, Sapena L, Ferrandis R. Perioperative management of antiplatelet agents in noncardiac surgery. Eur J Anaesthesiol 2009;26(3):181-187

19 Gregson J, Kaptoge S, Bolton T, et al; Emerging Risk Factors Collaboration. Cardiovascular risk factors associated with venous thromboembolism. JAMA Cardiol 2019;4(2):163-173

20 Wijeysundera DN, Wijeysundera HC, Yun L, et al. Risk of elective major noncardiac surgery after coronary stent insertion: a population-based study. Circulation 2012;126(11):1355-1362

21 Lindenauer PK, Pekow P, Wang K, Mamidi DK, Gutierrez B, Benjamin EM. Perioperative beta-blocker therapy and mortality after major noncardiac surgery. N Engl J Med 2005;353(4): 349-361

22 Bouri S, Shun-Shin MJ, Cole GD, Mayet J, Francis DP. Meta-analysis of secure randomised controlled trials of $\beta$-blockade to prevent perioperative death in non-cardiac surgery. Heart 2014;100(6):456-464

23 Beattie WS, Wijeysundera DN, Karkouti K, et al. Acute surgical anemia influences the cardioprotective effects of $\beta$-blockade: a single-center, propensity-matched cohort study. Anesthesiology 2010;112(1):25-33

24 Wallace AW, Au S, Cason BA. Association of the pattern of use of perioperative. $\beta$-blockade and postoperative mortality. Anesthesiology 2010;113(4):794-805

25 Lindenauer PK, Pekow P, Wang K, Gutierrez B, Benjamin EM. Lipid-lowering therapy and in-hospital mortality following major noncardiac surgery. JAMA 2004;291(17):2092-2099

26 Wiklund RA, Stein HD, Rosenbaum SH. Activities of daily living and cardiovascular complications following elective, noncardiac surgery. Yale J Biol Med 2001;74(2):75-87

27 Reilly DF, McNeely MJ, Doerner D, et al. Self-reported exercise tolerance and the risk of serious perioperative complications. Arch Intern Med 1999;159(18):2185-2192

28 Hlatky MA, Boineau RE, Higginbotham MB, et al. A brief self-administered questionnaire to determine functional capacity (the Duke Activity Status Index). Am J Cardiol 1989;64(10):651-654

29 Surgical Risk Calculator. Available at:http://www.surgicalriskcalculator.com/mior-cardiacarrest. Accessed January 11, 2019 
30 Fleisher LA, Beckman JA, Brown KA, et al; American College of Cardiology; American Heart Association Task Force on Practice Guidelines (writing Committee to Revise the 2002 Guidelines on Perioperative Cardiovascular Evaluation for Noncardiac Surgery); American Society of Echocardiography; American Society of Nuclear Cardiology; Heart Rhythm Society; Society of Cardiovascular Anesthesiologists; Society for Cardiovascular Angiography and Interventions; Society for Vascular Medicine and Biology; Society for Vascular Surgery. ACC/AHA 2007 guidelines on perioperative cardiovascular evaluation and care for noncardiac surgery: a report of the American College of Cardiology/American Heart Association Task Force on practice guidelines (Writing Committee to Revise the 2002 guidelines on perioperative cardiovascular evaluation for noncardiac surgery) developed in collaboration with the American Society of Echocardiography, American Society of Nuclear Cardiology, Heart Rhythm Society, Society of Cardiovascular Anesthesiologists, Society for Cardiovascular Angiography and Interventions, Society for Vascular Medicine and Biology, and Society for Vascular Surgery. J Am Coll Cardiol 2007;50(17):e159-e241

31 Frangiskakis JM, Hravnak M, Crago EA, et al. Ventricular arrhythmia risk after subarachnoid hemorrhage. Neurocrit Care 2009;10(3):287-294

32 Baumgartner H, Falk V, Bax JJ, et al; ESC Scientific Document Group. 2017 ESC/EACTS guidelines for the management of valvular heart disease. Eur Heart J 2017;38(36):2739-2791

33 Farzi S, Stojakovic T, Marko T, et al. Role of N-terminal pro B-type natriuretic peptide in identifying patients at high risk for adverse outcome after emergent non-cardiac surgery. Br J Anaesth 2013;110(4):554-560
34 Bastola P, Bhagat H, Wig J. Comparative evaluation of propofol, sevoflurane and desflurane for neuroanaesthesia: a prospective randomised study in patients undergoing elective supratentorial craniotomy. Indian J Anaesth 2015;59(5):287-294

35 Myburgh JA, Upton RN, Grant C, Martinez A. The effect of infusions of adrenaline, noradrenaline and dopamine on cerebral autoregulation under propofol anaesthesia in an ovine model. Intensive Care Med 2003;29(5):817-824

36 Jellinek D, Platt M, Jewkes D, Symon L. Effects of nitrous oxide on motor evoked potentials recorded from skeletal muscle in patients under total anesthesia with intravenously administered propofol. Neurosurgery 1991;29(4):558-562

37 Panah M, Kahn RA. Neurosurgery for the patient with cardiac disease. Anesthesiol Clin North Am 1997;15(1):159-170

38 Doshi R, Neil-Dwyer G. A clinicopathological study of patients following a subarachnoid hemorrhage. J Neurosurg 1980;52(3):295-301

39 Ward RJ, Danziger F, Bonica JJ, et al. Cardiovascular effects of change of posture. Aerosp Med 1966;37:257-259

40 Manikandan S. Dexmedetomidine: its fascination, fad, and facts in neuroanaesthesia practice! J Neuroanaesth Crit Care 2014;1:163-165

41 Tyler DS, Bacon D, Mahendru V, Lema MJ. Asystole as a neurologic sign. J Neurosurg Anesthesiol 1997;9(1):29-30

42 Chowdhury T, Petropolis A, Cappellani RB. Cardiac emergencies in neurosurgical patients. BioMed Res Int 2015;2015:751320 